EPJ Web of Conferences 19, 09008 (2012)

DOI: $10.1051 /$ epjconf/20121909008

(C) Owned by the authors, published by EDP Sciences, 2012

\title{
The AMBRE project: Results from the MATISSE analysis of the ESO-FEROS archived spectra
}

\author{
C.C. Worley ${ }^{\text {a }}$, P. de Laverny, A. Recio-Blanco, V. Hill and G. Kordopatis \\ Université de Nice Sophia Antipolis, CNRS (UMR 6202), Observatoire de la Côte D'Azur, \\ Cassiopée, BP.4229, 06304 Nice Cedex 04, France
}

\begin{abstract}
The goal of AMBRE, a joint project between ESO and the Observatoire de la Cote d'Azur, is to provide a homogeneous determination of the stellar parameters (including mean metallicity and some chemical abundances) for the archived spectra of the FEROS, HARPS, UVES and Flames/GIRAFFE spectrographs. We present here a preliminary analysis of the FEROS stellar sample. The stellar parameters, derived using the stellar classification algorithm MATISSE, have been combined with distances and radial velocities in order to explore current theories regarding galactic stellar populations. We have reviewed the sample for potential disk stars with which we can explore kinematic and chemical signatures of the thin and thick disk. We present here a comparison of the potential disk stellar sample to the analysis of 66 disk stars that was carried out in.
\end{abstract}

\section{INTRODUCTION}

Under a contract between the European Southern Observatory (ESO) and the Observatoire de la Cote d'Azur (OCA), the AMBRE Project is tasked with determining the stellar parameters for the archived spectra of four of ESO's spectrographs: FEROS, HARPS, UVES \& FLAMES/GIRAFFE, which will be made available to the astronomical community via the ESO Archive. The analysis of the FEROS spectra is now complete and the analysis of the UVES and HARPS spectra is underway.

This project presents a unique opportunity to exploit a large pre-existing spectral dataset $(\sim 250,000$ spectra) for global information outside the original goals of the individual observing programmes. Despite the inherent programme biases, the FEROS dataset (as for the remaining archived datasets) is a homogeneous determination of stellar parameters for a large spectral sample. An analysis pipeline has been built which futher treats the archived spectra in order that they may be fed into the stellar parametrisation algorithm, MATISSE, which has been developed at OCA [1]. An indepth discussion of the analysis pipeline and validation of the FEROS results will be presented in Worley et al. (2011), in preparation.

\section{VALIDATION WITH BENSBY ET AL (2003)}

An essential stage of the AMBRE Project has been the validation of the results from the analysis pipeline. Several spectral atlases (the Sun, Arcturus, Procyon) and spectral libraries (PASTEL [2], $\mathrm{S}^{4} \mathrm{~N}$ [3], Gaia RVS Standards [4]) have been exploited in an extensive testing and comparison process. A key study used for the comparison of stellar parameters, in particular for the $\alpha$ element abundances, was Bensby et al. (2003) (B03) [5]. This was a study of a sample of 66 F \& G dwarf stars in the Galactic

\footnotetext{
ae-mail: cworley@oca.eu
}

This is an Open Access article distributed under the terms of the Creative Commons Attribution-Noncommercial License 3.0, which permits unrestricted use, distribution, and reproduction in any noncommercial medium, provided the original work is properly cited. 

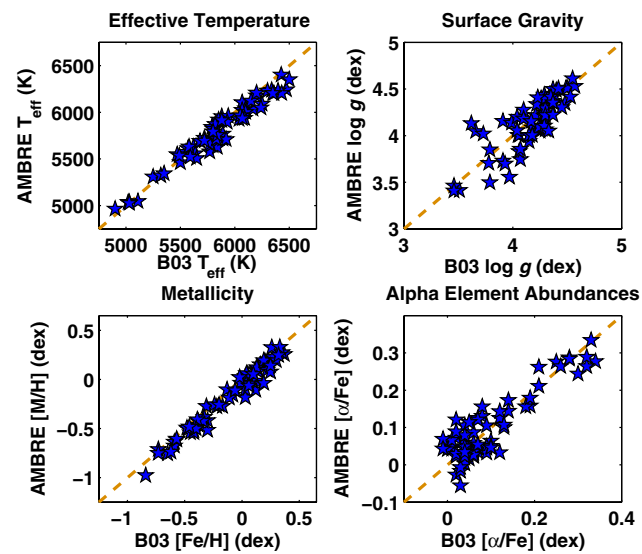

Figure 1. Comparison of the Bensby et al. (2003) 66 disk stars with the AMBRE:FEROS stellar parameters.

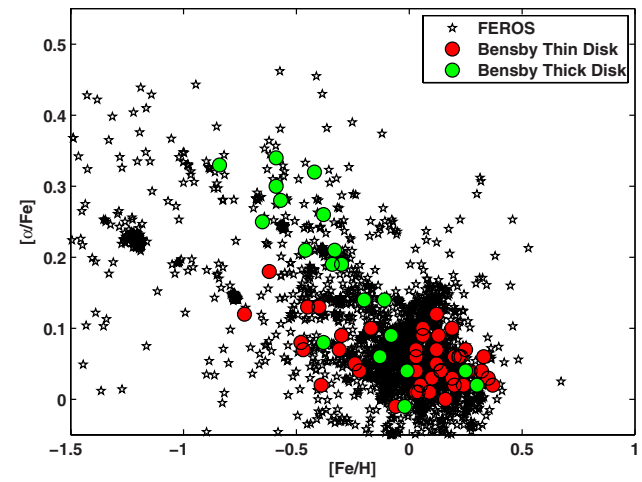

Figure 2. Distribution of $[\alpha / \mathrm{Fe}]$ with $[\mathrm{M} / \mathrm{H}]$ for 3342 of the FEROS spectra located in the disk compared to the Bensby et al. (2003) values.

disk that were observed using the FEROS spectrograph in 2000 and 2001. These same spectra were re-analysed as a test of the AMBRE analysis pipeline. Excellent agreement was found between the AMBRE stellar parameters and the literature values as shown in Figure 1.

\section{THICK \& THIN DISK STARS IN THE FEROS DATASET}

Of the 66 stars in the B03 sample, 21 were determined to be thick disk stars and 45 were determined to be thin disk stars. B03 carried out a detailed chemical abundance analysis of these spectra to distinguish thick and thin disk chemical signatures for these populations.

This sample was examined in a comparison between the AMBRE values and the $[\alpha / \mathrm{Fe}]$ and $[\mathrm{Fe} / \mathrm{H}]$ values determined in B03. Figure 2 shows the trend between these ratios for the FEROS stars compared with the B03 thick disk stars and the B03 thin disk stars. For the thick disk stars, the trend of high $[\alpha / \mathrm{Fe}]$ at low $[\mathrm{Fe} / \mathrm{H}]$ and the steep negative gradient in the near solar $[\mathrm{Fe} / \mathrm{H}]$ range is clearly replicated in the FEROS sample. This corresponds to the change in regime from Type II Supernova to Type 1a supernova dominating the star formation history of the thick disk [6]. The concentrated distribution of FEROS stars over the near solar $[\mathrm{Fe} / \mathrm{H}]$ range but only slightly enhanced $[\alpha / \mathrm{Fe}]$ is in good agreement with the B03 thin disk stars. 
Assembling the Puzzle of the Milky Way

\section{CONCLUSION}

The analysis of the FEROS archived spectra, in this first phase of the AMBRE Project, is now complete. The analyses of the UVES and HARPS archived datasets are now underway. The B03 reference sample clearly shows that this homogeneous determination of the stellar parameters of this large spectral dataset is in excellent agreement with other high quality high resolution spectroscopic studies. The analysis of the $\alpha$ and Fe element abundances shown here provides a significant extension to the study of thin and thick disk populations. This study is continuing and the sample is currently being analysed to determine the exact membership of these stars in either the thick or the thin disk using kinematic signatures only (Worley et al. 2012, in preparation). A full comparison of the $\alpha$ element and Fe abundance distributions for these populations within the FEROS sample will then be made.

\section{References}

[1] A. Recio-Blanco, A. Bijaoui, P. de Laverny, MNRAS 370, 141 (2006)

[2] C. Soubiran, J. Le Campion, G. Cayrel de Strobel, A. Caillo, A\&A 515, A111+ (2010), 1004. 1069

[3] C. Allende Prieto, P.S. Barklem, D.L. Lambert, K. Cunha, A\&A 420, 183 (2004)

[4] F. Crifo, G. Jasniewicz, C. Soubiran, D. Katz, A. Siebert, L. Veltz, S. Udry, A\&A 524, A10+ (2010)

[5] T. Bensby, S. Feltzing, I. Lundström, A\&A 410, 527 (2003)

[6] L.V. Sales, A. Helmi, M.G. Abadi, C.B. Brook, F.A. Gómez, R. Roškar, V.P. Debattista, E. House, M. Steinmetz, Á. Villalobos, MNRAS 400, L61 (2009) 